\title{
Validación de una estrategia didáctica gamificada para la enseñanza-aprendizaje de conceptos de ecología
}

\section{Validation of a gamified didactic strategy for the teaching-learning of ecology concepts}

\author{
NIÑO-VEGA, Jorge A. ${ }^{1}$ \\ DUCUARA-AMADO, Lydy Y.2 \\ FERNÁNDEZ-MORALES, Flavio $\mathrm{H}^{3}$
}

\begin{abstract}
Resumen
Se presenta una estrategia didáctica mediada por Tecnologías de la Información y la Comunicación (TIC) para la enseñanza de ecología, validada con 30 estudiantes de una institución educativa colombiana. La investigación fue cuantitativa, empleando estadística descriptiva para identificar el desempeño de los estudiantes, antes y después de implementar la estrategia didáctica. La diferencia en la media de las dos pruebas fue de 2,72. Se concluye que la estrategia didáctica influyó positivamente en el rendimiento de los estudiantes, gracias a que el material se diseñó con base en sus necesidades.

Palabras clave: estrategia didáctica, TIC, gamificación, ecología.
\end{abstract}

\begin{abstract}
An educational strategy mediated by Information and Communication Technologies (ICT) for teaching ecology is presented, validated with 30 students from a Colombian educational institution. The research was quantitative, using descriptive statistics to identify student performance, before and after implementing the teaching strategy. The difference in the mean of the two tests was 2.72 . It is concluded that the didactic strategy positively influenced the performance of the students, thanks to the fact that the material was designed based on their needs.

key words: didactic strategy, ICT, gamification, ecology.
\end{abstract}

\section{Introducción}

En la actualidad, el planeta afronta varias problemáticas medioambientales que amenazan con la existencia de los seres vivos (Ortiz-Villota, Romero-Morales \& Mesa-Rodríguez, 2018). Estas amenazas son consecuencia de factores antrópicos, tales como: la sobreexplotación de los recursos naturales, la deforestación y la contaminación, entre otros (Bautista-Ruiz, Díaz-Lagos \& Martínez-Ovalle, 2017). Algunas investigaciones destacan que estas acciones se deben al poco conocimiento que tienen las personas, sobre los impactos

\footnotetext{
${ }^{1}$ Docente investigador, Licenciado en Tecnología, Magíster en TIC Aplicadas a las Ciencias de la Educación, Universidad Pedagógica y Tecnológica de Colombia, Duitama, Colombia. E-mail: Jorge.ninovega@gmail.com |ORCID ID: https://orcid.org/0000-0001-7803-5535

${ }^{2}$ Docente, Licenciada en ciencias naturales y educación ambiental, Especialista en educación ambiental, Magíster en TIC Aplicadas a las Ciencias de la Educación, Universidad Pedagógica y Tecnológica de Colombia, Duitama, Colombia. E-mail: Iydy.ducuara@uptc.edu.co |ORCID ID: https://orcid.org/0000-0001-6172-9004

${ }^{3}$ Docente investigador, Ingeniero Electrónico, Doctor en Ingeniería Electrónica, Universidad Pedagógica y Tecnológica de Colombia, Duitama, Colombia. Email: flaviofm1@gmail.com |ORCID ID: https://orcid.org/0000-0002-8970-7146
} 
desfavorables que sus decisiones generan sobre el medio ambiente (Angarita-López, Duarte \& FernándezMorales, 2018; Lozano-Guzmán, Bosque-Suárez \& Osorio-Abad, 2019).

En este sentido, las instituciones educativas han venido orientando temáticas relacionadas con el cuidado y preservación de los ecosistemas acuáticos y terrestres, con el fin de que las personas se concienticen de la importancia de la fauna y la flora para la preservación del planeta (Aranda-Sánchez, 2015; Niño-Vega, FernándezMorales \& Duarte, 2019). Algunos docentes y ecologistas, preocupados por la preservación del medioambiente y conscientes de que la educación es un proceso continuo, que toma al estudiante no como objeto sino como un sujeto a quien se le debe enseñar para la vida, han desarrollado diversas estrategias pedagógicas para la enseñanza de la ecología (Carceller-Cobos, 2016; Hernández-Gil \& Jaramillo-Gaitán, 2020).

Estas estrategias, cada vez con mayor frecuencia, se encuentran mediadas por las Tecnologías de la Información y la Comunicación, TIC, las cuales se aplican en áreas del conocimiento tan disímiles como: física, matemáticas, medicina y administración, por mencionar algunas (Ruiz-Macías \& Duarte, 2018; Ordóñez-Ortega, GualdrónPinto \& Amaya-Franky, 2019; Niño-Vega \& Fernández-Morales, 2019; Espinosa-Urbina, 2019). Las TIC también han sido empleadas en los niveles de educación básica y media, para orientar conceptos relacionados con: el uso racional de la energía, el uso del recurso hídrico y prácticas sostenibles para la disposición final de las basuras, temáticas directamente involucradas con el cuidado y preservación del medio ambiente (Angarita-López et al., 2018; Niño-Vega et al., 2019; López-Pérez \& Guerrero-Erazo, 2017). En este sentido, las estrategias didácticas basadas en la gamificación, aprovechan el potencial de las TIC para incorporar las dinámicas del juego en el proceso formativo, empleando herramientas informáticas que despiertan la motivación de los nativos digitales por el aprendizaje de diversas temáticas (Ducuara-Amado et al., 2020; Iriarte-Pupo, 2020).

El Instituto Técnico Santo Tomás de Aquino, ITSTA, de la ciudad de Duitama, Colombia, no es ajeno a la problemática mencionada anteriormente. En esta institución, se enseña sobre ecología en el área de las ciencias naturales hasta educación básica, dejando como reemplazo en la educación media a la química. Esto ocasiona que los estudiantes no se responsabilicen de las consecuencias medioambientales de sus acciones, ni brinden soluciones para atenderlas. Además, la ausencia de estos conocimientos, hace que se reduzca el desempeño de los estudiantes en las pruebas de estado para quienes egresan de la educación media (Timarán-Pereira, CaicedoZambrano \& Hidalgo-Troya, 2019).

En este trabajo, se presenta la validación de una estrategia didáctica basada en TIC, para la orientación de conceptos de ecología en estudiantes de grado décimo, del ITSTA. A continuación, se reporta la metodología empleada para el desarrollo de la investigación, incluyendo el diseño didáctico aplicado en el aula. Luego se presentan los resultados de las pruebas inicial y final, se realiza el análisis de los datos y se brindan las conclusiones del estudio.

\section{Metodología}

Esta investigación se desarrolló bajo un enfoque cuantitativo, con alcance descriptivo, para determinar el efecto de una estrategia didáctica mediada por TIC, en el rendimiento académico de los estudiantes. Específicamente, se consideró la variable de rendimiento académico sobre conceptos de ecología, en estudiantes de grado décimo del ITSTA, antes y después de ser intervenidos con una estrategia basada en gamificación a través de TIC. Se utilizó un diseño cuasiexperimental, prueba inicial y prueba final con un solo grupo, puesto que no se tuvo la disponibilidad de la totalidad de la población objeto de estudio (Hernández-Sampieri, Fernández-Collado \& Baptista-Lucio, 2010).

La población objeto de estudio, correspondió a los 121 estudiantes del grado décimo del ITSTA. La muestra fue escogida por conveniencia debido a la disponibilidad de las salas de cómputo, y estuvo conformada por 30 
estudiantes del grado 1002, de la especialidad de diseño de modas. El trabajo investigativo se desarrolló durante el cuarto periodo académico del año 2019, en la asignatura de ciencias naturales, teniendo en cuenta las siguientes etapas:

Etapa 1. Prueba Inicial: inicialmente se aplicó una prueba que constó de 20 preguntas de selección múltiple, con única respuesta, adaptadas del cuadernillo de preguntas saber 11 (ICFES, 2018). Los resultados de dicha prueba, permitieron reconocer el conocimiento que traen los estudiantes sobre conceptos básicos de ecología. Posteriormente, se seleccionó el material didáctico a ser utilizado, con el fin de abordar las temáticas que presentaron mayor nivel de dificultad en los estudiantes (Ducuara-Amado et al., 2020).

Etapa 2. Experiencia de aula. En esta etapa se realizó la intervención pedagógica en el aula. En la figura 1 se presenta la estrategia didáctica mediada por TIC, planteada en esta investigación.

Figura 1

Diseño didáctico propuesto.

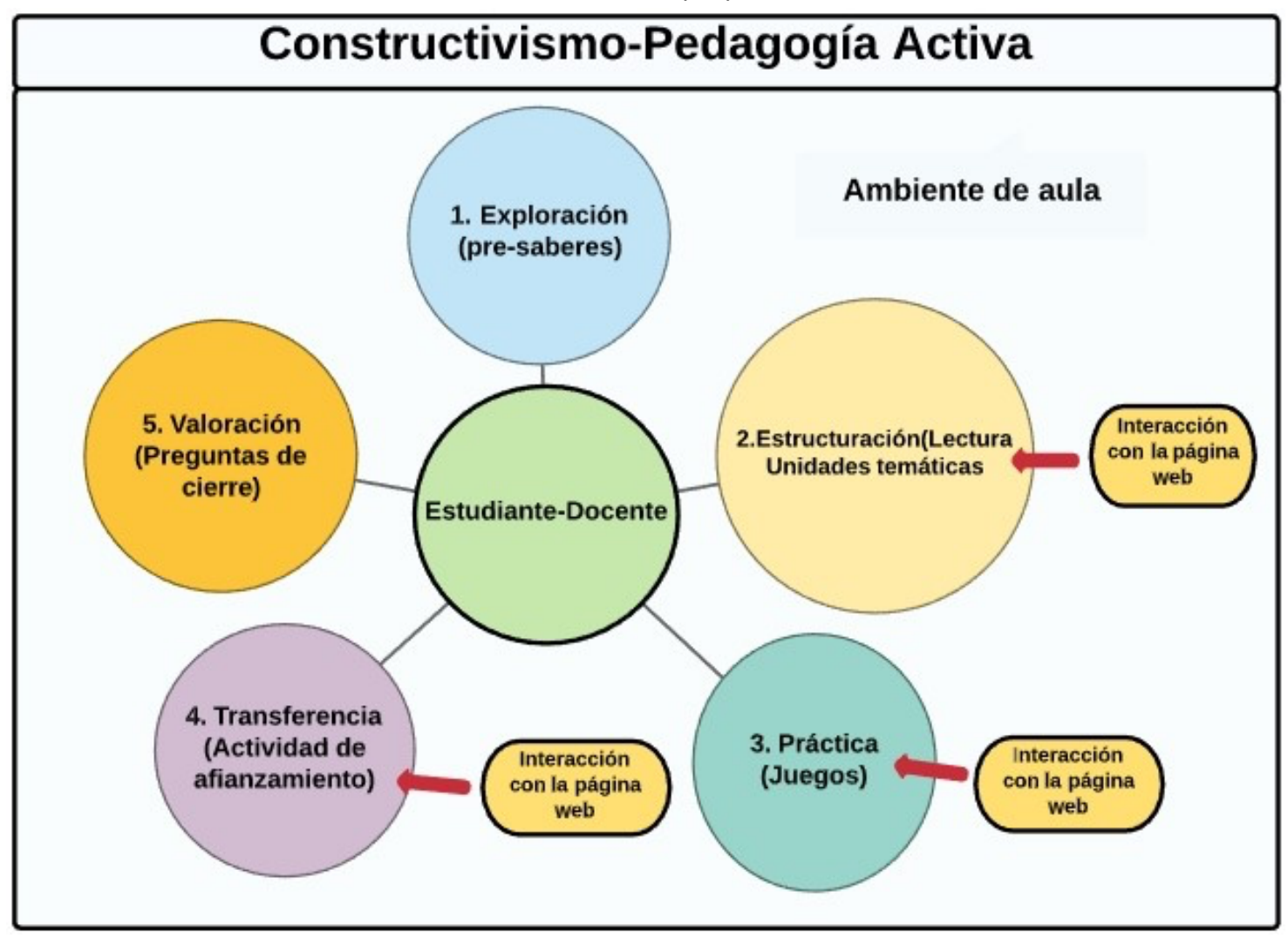

Fuente: Elaboración propia

Se resalta como centro del proceso formativo al estudiante, quien es acompañado por la orientación del docente, todo ello enmarcado desde la metodología del constructivismo y la pedagogía activa (Garzón-Saladen \& RomeroGonzález, 2018), que son la base del modelo pedagógico que maneja la institución educativa.

En las fases, se propone que el estudiante explore los contenidos que se encuentran inmersos en las diferentes unidades alojadas en el Recurso Educativo Digital, RED denominado: "Ecología y gamificación: por un mejor planeta", que está disponible en el sitio Web: https://ecologygamificacio.wixsite.com/2019/home.

Luego, se establece un espacio para que el estudiante ponga en práctica sus conocimientos, a través de videojuegos educativos. Seguidamente, se plantean actividades que permiten retroalimentar y evaluar los 
conceptos vistos en cada unidad. Por último, se hace una valoración de las unidades a través de foros de discusión, donde el estudiante plantea soluciones lógicas ante los problemas medioambientales que se enuncian.

Como actividad final de la intervención pedagógica, se realizó una prueba final, con el fin de identificar los conocimientos que adquirieron los estudiantes con respecto a conceptos de ecología. Las preguntas fueron similares a las de la prueba inicial, y los niveles de desempeño fueron valorados así: Bajo (1.0 -5.9), Básico (6.7.5), Alto (7.6 - 9.0), y Superior (9.1 - 10.0).

Etapa 3. Validación de la estrategia didáctica. En esta etapa se realizó un análisis estadístico para comprobar el nivel de efectividad de la estrategia didáctica propuesta. El procesamiento de la información se realizó a través de Excel de Microsoft Office, para la organización de los datos, mientras que para el análisis de la variable rendimiento académico se manejó el software de acceso libre "R Studio" (Ruiz-Macías, Duarte \& FernándezMorales, 2018).

Se analizó la variable puntaje de las pruebas inicial y final, para determinar el desempeño de los educandos después de la aplicación de la estrategia mediada por TIC. En este sentido, se realizó un análisis de asociación entre las pruebas utilizando tablas de contingencia para observar la diferencia de los desempeños obtenidos. Asimismo, se realizó el modelamiento de las pruebas utilizando el test de Shapiro Wilk, junto con el análisis de varianza unifactorial (ANOVA) para identificar la distribución de los datos y la ganancia de aprendizaje. Adicionalmente, se efectuó un contraste estadístico Chi-cuadrado, para establecer si la estrategia didáctica tuvo efecto sobre el rendimiento de los estudiantes en la prueba final.

\section{Resultados y discusión}

\subsection{Prueba inicial}

Como aspecto inicial del estudio, se procedió a identificar los conocimientos previos que tenían los estudiantes, referentes a conceptos básicos de ecología. Para ello, se aplicó un cuestionario de 20 preguntas, distribuidas así: 5 alusivas a ¿qué es un ecosistema?, factores abióticos y bióticos; 5 relacionadas con tipos de ecosistemas, acuáticos y terrestres; 5 preguntas referentes a relaciones ecológicas intraespecíficas e interespecíficas; y 5 preguntas entorno a flujo de materia y energía en los ecosistemas, y estructura trófica de los ecosistemas. En la tabla 1 , se presentan los resultados de la prueba inicial.

Tabla 1

Desempeños obtenidos por los estudiantes en la prueba inicial.

\begin{tabular}{|l|c|c|c|c|}
\hline \multicolumn{1}{|c|}{ Ítem } & \multicolumn{4}{c|}{ Desempeño } \\
\cline { 2 - 5 } & Bajo & Básico & Alto & Superior \\
\hline ¿Qué es un ecosistema?, factores abióticos y bióticos. & 19 & 10 & 1 & 0 \\
\hline Tipos de ecosistemas: acuáticos y terrestres. & 15 & 12 & 2 & 1 \\
\hline $\begin{array}{l}\text { Relaciones ecológicas intraespecíficas e } \\
\text { interespecíficas. }\end{array}$ & 14 & 16 & 0 & 0 \\
\hline $\begin{array}{l}\text { Flujo de materia y energía en los ecosistemas y } \\
\text { estructura trófica de los ecosistemas. }\end{array}$ & 12 & 15 & 1 & 2 \\
\hline
\end{tabular}

Fuente: Elaboración propia

En la tabla 1, se observa que tan solo 2 estudiantes obtuvieron un desempeño superior, en cuanto a la identificación de flujo de materia y energía en los ecosistemas. Igualmente, se muestra que tan solo 2 estudiantes obtuvieron un desempeño alto en cuanto al reconocimiento de los tipos de ecosistemas acuáticos y terrestres. Asimismo, el desempeño básico fue alcanzado por 16 estudiantes en cuanto a las relaciones ecológicas 
intraespecíficas e interespecíficas. Por último, se evidencia que la mayoría de estudiantes (19), obtuvieron un nivel de desempeño bajo, frente al ítem: ¿Qué es un ecosistema?, factores abióticos y bióticos.

Los resultados anteriores permitieron identificar que los estudiantes presentan dificultad para reconocer algunos conceptos básicos de ecología. Esto reforzó la necesidad de aplicar una estrategia pedagógica, que permita enseñar a los estudiantes sobre ecología y medioambiente. La estrategia seleccionada se basa en la gamificación para la enseñanza de conceptos de ecología, a través de herramientas propias de las TIC.

\subsection{Experiencia de aula}

Una vez identificadas las temáticas que presentan falencias de aprendizaje por parte de los estudiantes, y establecido el material educativo a ser utilizado, se procedió a implementar la estrategia pedagógica con los estudiantes de grado décimo. El trabajo de aula se llevó a cabo en 5 sesiones, con 2 horas presenciales por sesión. A continuación, se hace una breve descripción de las actividades realizadas:

En la sesión 1, la temática desarrollada fue: ¿qué es un ecosistema?. En este caso, el objetivo de aprendizaje fueron los conceptos de: ecosistema, factores bióticos y abióticos.

En la sesión 2, se abordó la temática denominada Tipos de Ecosistemas, que tuvo por objetivo comprender que dependiendo del medio en el que se desarrollan los seres vivos, existen ecosistemas terrestres y acuáticos.

En la sesión 3, la temática desarrollada fue Tipos de Ecosistemas Colombianos. El objetivo de esta sesión, fue el de valorar la diversidad de los ecosistemas colombianos, resaltando los impactos favorables y desfavorables que el hombre genera para la preservación o deterioro de los ecosistemas.

En la sesión 4, la temática abordada fue la de relaciones ecológicas. Esta tuvo como objetivo el identificar los tipos de relaciones intraespecíficas e interespecíficas, para que los estudiantes reconocieran como se establecen las especies dentro de un ecosistema.

En la quinta sesión, se desarrolló la temática Flujo de materia y energía en los Ecosistemas. El objetivo de esta sesión, fue el de explicar las relaciones entre materia y energía en las cadenas alimentarias.

Todas las sesiones se desarrollaron en tres momentos, así: inicialmente, los estudiantes exploraban el contenido temático de la sesión, disponible en el RED utilizado.

En el segundo momento, los estudiantes accedían a los juegos propuestos en cada unidad, cuyo propósito era poner en práctica el conocimiento adquirido, retroalimentándolo a través del error. Para ello, el estudiante disponía de: juegos en línea con límite de tiempo, juegos de preguntas y respuestas, actividades de emparejamiento, juegos de aventuras y de obstáculos.

El tercer momento consistió en el desarrollo de una actividad de aprendizaje, a partir de material complementario disponible en el RED. La intencionalidad de este momento, era que los estudiantes aplicaran sus saberes para sensibilizar a los demás sobre el cuidado y protección de los ecosistemas. En este sentido, se elaboraron materiales como: posters, folletos y carteleras, al igual que presentaciones hechas con herramientas digitales, tales como: canva, genialy y GoCongr.

Luego de la intervención pedagógica, se procedió a aplicar una prueba final para identificar los conocimientos sobre ecología que adquirieron los estudiantes. En este caso, se evaluaron los mismos criterios de la prueba inicial, pero con diferentes preguntas. En la tabla 2, se ilustran los desempeños obtenidos en la prueba final. 
Tabla 2

Desempeños obtenidos por los

estudiantes en la prueba inicial.

\begin{tabular}{|c|c|c|c|c|}
\hline \multirow{2}{*}{ Ítem } & \multicolumn{4}{|c|}{ Desempeño } \\
\hline & Bajo & Básico & Alto & Superior \\
\hline ¿Qué es un ecosistema?, factores abióticos y bióticos. & 3 & 8 & 9 & 10 \\
\hline Tipos de ecosistemas: acuáticos y terrestres. & 2 & 4 & 9 & 15 \\
\hline $\begin{array}{l}\text { Relaciones ecológicas intraespecíficas e } \\
\text { interespecíficas. }\end{array}$ & 1 & 14 & 8 & 7 \\
\hline $\begin{array}{l}\text { Flujo de materia y energía en los ecosistemas y } \\
\text { estructura trófica de los ecosistemas. }\end{array}$ & 2 & 9 & 14 & 5 \\
\hline
\end{tabular}

Fuente: Elaboración propia

Los resultados de la prueba final, muestran que: tan solo 3 estudiantes se encuentran en un nivel bajo, en cuanto a ¿Qué es un ecosistema?, factores abióticos y bióticos. Igualmente, 14 estudiantes obtuvieron un desempeño básico, en el criterio de Relaciones ecológicas intraespecíficas e interespecíficas. Además, el desempeño alto fue alcanzado por 14 estudiantes, en la temática de: Flujo de materia y energía en los ecosistemas y estructura trófica de los ecosistemas. Por último, se puede evidenciar que la mayoría de estudiantes (15), obtuvieron un nivel de desempeño superior frente al tema de tipos de ecosistemas: acuáticos y terrestres.

\subsection{Validación de la estrategia mediada por TIC}

Para validar el efecto que tuvo la estrategia pedagógica implementada en este estudio, se realizó un tratamiento estadístico de los resultados obtenidos en las pruebas inicial y final. En la tabla 3, se presenta una tabla de contingencia que relaciona el número de estudiantes, según el nivel de desempeño alcanzado en cada prueba.

Tabla 3

Desempeños obtenidos por los

estudiantes en la prueba inicial.

\begin{tabular}{|c|c|c|c|c|}
\hline \multirow{2}{*}{ Tipo de prueba } & \multicolumn{4}{|c|}{ Desempeño } \\
\cline { 2 - 5 } & Bajo & Básico & Alto & Superior \\
\hline Inicial & 15 & 13 & 1 & 1 \\
\hline Final & 2 & 9 & 10 & 9 \\
\hline
\end{tabular}

Fuente: Elaboración propia

Los resultados de la tabla 3, evidencian una mejora considerable en los desempeños, debido a que luego de haber tan solo 1 estudiante en el nivel alto en la prueba inicial, esta cantidad se incrementó a 10 estudiantes en la prueba final. Igualmente, el número de estudiantes en nivel superior, pasó de 1 en la prueba inicial, a 9 en la prueba final. En contraste, el desempeño bajo se redujo de 15 estudiantes en la prueba inicial, a tan solo 2 en la prueba final.

Para determinar si las puntuaciones obtenidas por los estudiantes en las pruebas inicial y final, siguen una distribución normal, se utilizó el test de Shapiro Wilk con un valor de confianza del 95\%. En este caso, se formuló como hipótesis nula: "la distribución es normal", y como hipótesis alterna: "la distribución no es normal". Al aplicar el test de Shapiro Wilk en el software R studio, se obtuvo un p-valor de 0.2048: debido a que el p-valor obtenido es mayor a 0.05, se acepta la hipótesis nula. Es decir, que hubo normalidad en la distribución de los datos analizados.

En vista de lo anterior, se procedió a validar si la diferencia de medias entre las pruebas inicial y final, es significativa. Para ello, se aplicó un análisis de varianza unifactorial, ANOVA, tomando como hipótesis nula que: 
"las medias de las pruebas inicial y final son iguales", mientras que la hipótesis alterna fue: "las medias de las pruebas inicial y final son diferentes". En los cálculos se tuvo en cuenta un nivel de significancia del 95\%.

Al aplicar el ANOVA, se obtuvo un p-valor de 0.000164, y debido a que este valor es menor a 0.05 , se rechaza la hipótesis nula. Por tanto, se puede decir que las medias de las pruebas inicial y final son diferentes. La media obtenida por los estudiantes en la prueba inicial fue de 5.33, mientras que en la prueba final fue de 8.05. Esto indica que existe una diferencia de 2.72 en las medias obtenidas por los estudiantes.

Adicionalmente, con el fin de identificar si la estrategia didáctica implementada tuvo efecto sobre los desempeños obtenidos por los estudiantes en la prueba final, se realizó el contraste estadistico chi-cuadrado. En este caso, se estableció como hipótesis nula: "el resultado de la prueba final es independiente a la implementación de la estrategia", y como hipótesis alterna: "el resultado de la prueba final no es independiente a la implementación de la estrategia". El resultado de la prueba chi-cuadrado, arrojó un P-Valor=0.0004508. Teniendo en cuenta que este valor es inferior a 0.05, se evidencia que la estrategia mediada por TIC si influyó en el desempeño de los estudiantes en la prueba final.

\subsection{Discusión}

El análisis estadístico de la sección anterior, permitió comprobar que los estudiantes mejoraron considerablemente su rendimiento académico en cuanto a conceptos de ecología. Esto se evidencia en que en la prueba inicial tan solo aprobaron el 50\% (15 estudiantes), mientras que en la prueba final aprobó el 93.3\% ( 28 estudiantes). Lo anterior indica que la estrategia didáctica mediada por TIC para la enseñanza-aprendizaje de conceptos de ecología, fue efectiva gracias a la estructuración de los contenidos, así como al material educativo utilizado, que se elaboró a partir de las necesidades que presentaron los estudiantes.

En cuanto a la implementación de la estrategia didáctica en el aula, se observó que los estudiantes participaron activamente en el desarrollo de las actividades propuestas. Igualmente, se evidenció una motivación y deseo por superar cada una de las actividades gamificadas, incluidas en las unidades temáticas, situación reportada en investigaciones similares (Avella-Ibáñez, Sandoval-Valero \& Montañez-Torres, 2017; Niño-Vega et al., 2017). Esto indica que, aunque los contenidos sean difíciles de comprender por parte de los estudiantes y difíciles de explicar por parte de los docentes, si se cuenta con el material adecuado, se vinculan las nuevas tecnologías, y se establece un ambiente de aprendizaje bien diseñado, será posible superar estas vicisitudes (Barrera-Mesa, Fernández- Morales \& Duarte, 2018; Bernate et al., 2020; Martínez-López \& Gualdrón-Pinto, 2018).

En cuanto a las TIC integradas a la educación, en esta investigación se evidenció que su utilización es pertinente para la enseñanza de conceptos de ecología, en el nivel de educación media. En este sentido, los hallazgos anteriores van en la línea de considerar que las TIC tienen un gran futuro en el sector educativo (GutiérrezRodríguez, 2018), ya que son facilitadores del proceso de enseñanza-aprendizaje (Ñáñez-Rodríguez, SolanoGuerrero \& Bernal-Castillo, 2019; Clavijo-Cáceres, 2018). Asimismo, las TIC permiten: generar cultura ciudadana (Angarita-López et al., 2018); desarrollar competencias y habilidades (Fonseca-Barrera, Niño-Vega, \& FernándezMorales, 2020; Vargas-Vargas, Niño-Vega \& Fernández-Morales, 2020); y, sobre todo, permiten que la educación sea accesible e inclusiva, gracias a las múltiples herramientas digitales y tecnológicas de las que se dispone (Angarita-López et al., 2020; Niño-Vega, Morán-Borbor \& Fernández- Morales, 2018).

Finalmente, se puede decir que no basta con diseñar materiales educativos, recursos digitales en este caso, para la enseñanza-aprendizaje de una temática específica. Sino que también es importante validarlos desde dos perspectivas: la opinión de los expertos y usuarios, así como la comprobación de la ganancia de aprendizaje que se genera al interactuar con dicho material educativo (Barrera-Mesa, Fernández-Morales \& Duarte, 2017; LópezGaitán, Moran-Borbor \& Niño-Vega, 2018). 


\section{Conclusiones}

En esta investigación se validó una estrategia didáctica mediada por TIC, complementada con un material didáctico gamificado, orientado a la enseñanza-aprendizaje de: ecosistemas acuáticos y terrestres, factores bióticos y abióticos, relaciones ecológicas y flujo de materia y energía en los ecosistemas.

El análisis estadístico de los desempeños obtenidos en las pruebas inicial y final, evidenciaron mejor rendimiento de los estudiantes en la prueba final. Esto permite concluir que la estrategia mediada por TIC, influyó positivamente en el aprendizaje de conceptos básicos de ecología.

La intervención pedagógica para la implementación de la estrategia con la población objeto de estudio, se adelantó bajo un modelo constructivista. Este hecho fue positivo, pues los estudiantes mostraron gran interés en el desarrollo de las actividades propuestas, especialmente en las gamificadas, lo que indica la necesidad de incorporar modelos pedagógicos donde el estudiante sea el protagonista de su propio aprendizaje.

En síntesis, los resultados de esta investigación, permiten afirmar que las TIC aplicadas a la enseñanza de la ecología, son una alternativa plausible para ser integradas en el aula. No obstante, el éxito de emplear nuevas tecnologías y nuevas estrategias pedagógicas, dependerá del diagnóstico que cada docente realice para seleccionar las herramientas más adecuadas a su contexto.

\section{Referencias bibliográficas}

Angarita-López, R. D., Duarte, J. E., \& Fernández-Morales, F. H. (2018). Desarrollo de un MEC para la creación de cultura ciudadana sobre el uso del recurso hídrico en estudiantes de educación básica. Revista Espacios, 39(15), 19. Recuperado de: https://www.revistaespacios.com/a18v39n15/18391519.html

Angarita-López, R. D., Fernández-Morales, F. H., Niño-Vega, J. A., Duarte, J. E., Gutiérrez-Barrios, G. J. (2020). Accesibilidad de las revistas colombianas del área de humanidades bajo las pautas WCAG 2.1. Revista Espacios, 41(4), 18. Recuperado de: http://revistaespacios.com/a20v41n04/20410418.html

Aranda-Sánchez, J. M. (2015). La alfabetización ecológica como nueva pedagogía para la comprensión de los seres vivientes. Luna Azul, (41), 365-384. Recuperado de:

http://www.scielo.org.co/pdf/luaz/n41/n41a20.pdf

Avella-Ibáñez, C. P., Sandoval-Valero, E. M., \& Montañez-Torres, C. (2017). Selección de herramientas web para la creación de actividades de aprendizaje en Cibermutua. Revista de investigación, Desarrollo e Innovación, 8(1), 107-120. doi: https://doi.org/10.19053/20278306.v8.n1.2017.7372

Barrera-Mesa, C. E., Fernández-Morales, F. H., \& Duarte, J. E. (2018). Validación de un ambiente de aprendizaje para la enseñanza de operadores mecánicos en educación básica. Revista Espacios, 39(25), 2. Recuperado de: http://www.revistaespacios.com/a18v39n25/18392502.html

Barrera-Mesa, M., Fernández-Morales, F. H., \& Duarte, J. E. (2017). Aprendizaje basado en proyectos colaborativos mediados por TIC para el desarrollo de competencias en estadística. Saber, Ciencia y Libertad, 12 (2), 220-232. Recuperado de:

http://www.sabercienciaylibertad.org/ojs/index.php/scyl/article/view/247

Bautista-Ruiz, W., Díaz-Lagos, M., \& Martínez-Ovalle, S. (2017). Caracterización de las cenizas volantes de una planta termoeléctrica para su posible uso como aditivo en la fabricación de cemento. Revista de Investigación, Desarrollo e Innovación, 8(1), 135-146. doi:

https://doi.org/10.19053/20278306.v8.n1.2017.7374 
Bernate, J. A., García-Celis, M. F., Fonseca-Franco, I. P., \& Ramírez-Ramírez- N. E. (2020). Prácticas de enseñanza y evaluación en una facultad de educación colombiana. Revista de Investigación, Desarrollo e Innovación, 10 (2), 337-347. doi: https://doi.org/10.19053/20278306.v10.n2.2020.10721

Carceller-Cobos, C. (2016). La gamificación en aplicaciones móviles ecológicas: análisis de componentes y elementos de juego. Sphera Publica, 1(16), 95-113. Recuperado de: http://193.147.26.137/index.php/sphera-01/article/view/280/249

Clavijo-Cáceres, D. (2018). Competencias del docente universitario en el siglo XXI. Revista Espacios, 39 (20), 22. Recuperado de: http://www.revistaespacios.com/a18v39n20/18392022.html

Ducuara-Amado, L. Y., Rodríguez-Hernández, A. A., Niño-Vega, J. A., \& Fernández-Morales, F. H. (2020). Material educativo gamificado para la enseñanza-aprendizaje de conceptos de ecología en estudiantes de educación media. Revista Boletín Redipe, 9 (6), 144-156. Doi: https://doi.org/10.36260/rbr.v9i6.1008

Espinosa-Urbina, G. B. (2019). Estrategia pedagógica basada en las tecnologías de información y comunicación (tic), para la enseñanza de los principios básicos de la convivencia social. Aibi Revista De investigación, administración E ingeniería, 7(2), 33-37. Doi: https://doi.org/10.15649/2346030X.567

Fonseca-Barrera, C. C., Niño-Vega, J. A., \& Fernández-Morales, F. H. (2020). Desarrollo de competencias digitales en programación de aplicaciones móviles en estudiantes de noveno grado a través de tres estrategias pedagógicas. Revista Boletín Redipe, 9(4), 179-191. Doi: https://doi.org/10.36260/rbr.v9i4.958

Garzón-Saladen, Á., \& Romero-González, Z. (2018). Los modelos pedagógicos y su relación con las concepciones del derecho: puntos de encuentro con la educación en derecho. Revista de Investigación, Desarrollo e Innovación, 8(2), 311-320. doi: https://doi.org/10.19053/20278306.v8.n2.2018.7968

Gutiérrez-Rodríguez, C. (2018). Fortalecimiento de las competencias de interpretación y solución de problemas mediante un entorno virtual de aprendizaje. Revista de Investigación, Desarrollo e Innovación, 8(2), 279293. doi: https://doi.org/10.19053/20278306.v8.n2.2018.7170

Hernández-Gil, C., \& Jaramillo-Gaitán, F. A. (2020). Laboratorio de innovación social: hibridación creativa entre las necesidades sociales y las experiencias significativas de los estudiantes de administración de empresas. Revista de Investigación, Desarrollo e Innovación, 10 (2), 267-281. doi:

10.19053/20278306.v10.n2.2020.10518

Hernández-Sampieri, R., Fernández-Collado, C., \& Baptista-Lucio, P. (2010). Metodología de la Investigación. Chile: McGraw Hill.

ICFES (2018). Cuadernillo de preguntas Saber 11. ${ }^{\circ}$ : prueba de ciencias naturales. Recuperado de: https://www.icfes.gov.co/documents/20143/1156910/Cuadernillo\%20de-preguntas-Saber-11-cienciasnaturales.pdf

Iriarte-Pupo, A. (2020). Fenomenología-hermenéutica de la investigación formativa. El formador de formadores: de la imposición a la transformación. Revista de Investigación, Desarrollo e Innovación, 10(2), 311-322. doi: https://doi.org/10.19053/20278306.v10.n2.2020.10722

López-Gaitán, M. A., Moran-Borbor, R. A., \& Niño-Vega, J. A. (2018). Prácticas experimentales como estrategia didáctica para la comprensión de conceptos de física mecánica en estudiantes de educación superior. Infometric@ - Serie Ingeniería, Básicas y Agrícolas, 1 (1), 1-14. Recuperado de: http://infometrica.org/index.php/syh/article/view/12 
López-Pérez, F., \& Guerrero-Erazo, J. (2017). Consideraciones ambientales sobre las prácticas de consumo de agua y energía en hogares urbanos. Revista Espacios, 38 (59), 28. Recuperado de:

http://www.revistaespacios.com/a17v38n59/17385928.html

Lozano-Guzmán, S. L., Bosque-Suárez, R., \& Osorio-Abad, A. (2019). La educación ambiental en el marco de la revolución científico-técnica: una necesidad actual en el contexto educativo colombiano. Varona. Revista Científico Metodológica, (68), e22. Recuperado de:

http://scielo.sld.cu/scielo.php?script=sci_arttext\&pid=S1992-82382019000100022\&lng=es\&tlng=es.

Martínez-López, L. G., \& Gualdrón-Pinto, E. (2018). Fortalecimiento del pensamiento variacional a través de una intervención mediada con TIC en estudiantes de grado noveno. Revista de Investigación, Desarrollo e Innovación, 9(1), 91-102. doi: https://doi.org/10.19053/20278306.v9.n1.2018.8156

Niño-Vega, J. A., \& Fernández- Morales, F. H. (2019). Una mirada a la enseñanza de conceptos científicos y tecnológicos a través del material didáctico utilizado. Revista Espacios, 40 (15). Recuperado de: http://www.revistaespacios.com/a19v40n15/19401504.html

Niño-Vega, J. A., Fernández-Morales, F. H., \& Duarte, J. E. (2019). Diseño de un recurso educativo digital para fomentar el uso racional de la energía eléctrica en comunidades rurales. Saber, Ciencia y Libertad, 14 (2), 256-272. Doi: https://doi.org/10.18041/2382-3240/saber.2019v14n2.5889

Niño-Vega, J. A., Martínez-Díaz, L. Y., Fernández-Morales, F. H., Duarte, J. E., Reyes-Caballero, F., \& GutiérrezBarrios, G. J. (2017). Entorno de aprendizaje para la enseñanza de programación en Arduino mediado por una mano robótica didáctica. Revista Espacios, 38 (60), 23. Recuperado de: http://www.revistaespacios.com/a17v38n60/17386023.html

Niño-Vega, J. A., Morán-Borbor, R. A., \& Fernández-Morales, F. H. (2018). Educación inclusiva: un nuevo reto para la labor docente en el siglo XXI. Infometric@ - Serie Sociales y Humanas, 1 (2), 74-94. Recuperado de: http://www.infometrica.org/index.php/ssh/article/view/78

Ñáñez-Rodríguez, J., Solano-Guerrero, J., \& Bernal-Castillo, E. (2019). Ambientes digitales de aprendizaje en educación a distancia para la formación inicial de docentes: percepciones acerca de su pertinencia. Revista de Investigación, Desarrollo e Innovación, 10(1), 107-119. Doi:

https://doi.org/10.19053/20278306.v10.n1.2019.10015

Ordóñez-Ortega, O., Gualdrón-Pinto, E., \& Amaya-Franky, G. (2019). Pensamiento variacional mediado con baldosas algebraicas y manipuladores virtuales. Revista de Investigación, Desarrollo e Innovación, 9 (2), 347-362. doi: https://doi.org/10.19053/20278306.v9.n2.2019.9180

Ortiz-Villota, M. T., Romero-Morales, M. A., \& Meza-Rodríguez, L. D. (2018). La biorremediación con microalgas (Spirulina máxima, Spirulina plantensis y Chlorella vulgaris) como alternativa para tratar la eutrofización de la laguna de Ubaque, Colombia. Revista de Investigación, Desarrollo e Innovación, 9 (1), 163-176. doi: https://doi.org/10.19053/20278306.v9.n1.2018.8153

Ruiz-Macías, E., \& Duarte, J. E. (2018). Diseño de un material didáctico computarizado para la enseñanza de oscilaciones y ondas, a partir del estilo de aprendizaje de los estudiantes. Revista de Investigación, Desarrollo e Innovación, 8(2), 295-309. doi: https://doi.org/10.19053/20278306.v8.n2.2018.7966

Ruiz-Macías, E., Duarte, J. E., \& Fernández-Morales, F. H. (2018). Validación de un material didáctico computarizado para la enseñanza de Oscilaciones y Ondas a partir del estilo de aprendizaje de los 
estudiantes. Revista Espacios, 39 (49), 38. Recuperado de:

http://www.revistaespacios.com/a18v39n49/18394938.html

Timarán-Pereira, R., Caicedo-Zambrano, J., \& Hidalgo-Troya, A. (2019). Árboles de decisiones para predecir factores asociados al desempeño académico de estudiantes de bachillerato en las pruebas saber $11^{\circ}$.

Revista de Investigación, Desarrollo e Innovación, 9 (2), 363-378. doi:

https://doi.org/10.19053/20278306.v9.n2.2019.9184

Vargas-Vargas, N. A., Niño-Vega, J. A., \& Fernández-Morales, F. H. (2020). Aprendizaje basado en proyectos mediados por tic para superar dificultades en el aprendizaje de operaciones básicas matemáticas. Revista Boletín Redipe, 9(3), 167-180. Doi: https://doi.org/10.36260/rbr.v9i3.943

Esta obra está bajo una Licencia Creative Commons Attribución-NoCommercial 4.0 International

(cc) BY-NC 